\title{
Der Haut=Haarwurzel=Winkel, die Haarwurzellänge und Ansatzhöhe des Haarbalgmuskels am Haarbalg bei dem japanischen Neugeborenen.
}

\author{
Von \\ Susumu Koibuchi. \\ Aus dem Anatomischen Institut der Keio Universität, Tokyo. \\ Mit 4 Textfiguren.
}

\section{Einleitung.}

Über die Haarrichtung und den Haarstrich haben seit geraumer Zeit viele Autoren sehr eingehende Studien gemacht. Aber der Winkel, der zwischen der Haarwurzel und Hautoberfläche entsteht (Haut-Haarwurzel-Winkel) und für die Bildung der Haarrichtung und des Haarstriches ein wichtiges Moment gibt, ist bis jetzt noch unaufgeklärt ${ }^{1}$; besonders ist der betreffende Winkel in verschiedenen Körperteilen beim Neugeborenen von niemandem verfolgt worden. Ferner sind die Länge der Haarwurzel und die Höhe der Ansatzstelle des Arrektors am Haarbalg beim Erwachsenen und bei Säugetieren auch von einigen Autoren (Auburtin (4), Bonnet (1), Häggqvist (5), Kanaizuka (6), Rabl (11), Unna (12) u. a.) berechnet worden; aber ihre Angaben beruhen nur auf der einfachen Betrachtung an den senkrechten und Flächenschnitten der Haut oder an den Wachsplattenmodellen und sind auch nur an wenigen Körperteilen zu sehr beschränkten Ergebnissen gekommen.

In der vorliegenden Untersuchung habe ich die obigen Probleme an den Serienschnitten der Haut von 11 verschiedenen Körperteilen bei

1) Der Winkel zwischen dem Haarschaft und der Hautoberfläche wurde von Basler (2) gemessen und auch der betreffende Winkel der Kopfhare der verschiedenen Menschenrassen wurde von Frédéric (3) an den mikroskopischen Präparaten berechnet. 
einem japauischen Neugeborenen nach der mikrogeometrischen Methode von Okajima (9) verfolgt und konnte folgendes Resultat gewinnen.

\section{Material und Methode.}

Das mir zur Verfügung gestellte Material stammt aus einem neugeborenen japanischen Kind (Körpergewicht $2.400 \mathrm{~kg}$, Körperlänge $152 \mathrm{~mm}$, o ), das nach dem Tode sogleich mit Formol-Alkohol-Lösung allgemein injiziert wurde und dann in 10\% Formalinlösung kam, worin es ca. ein Jahr lang konserviert wurde, um die Schrumpfung der herauszuschneidenden Haut $\mathrm{zu}$ vermeiden. Die Hautstücke von ca. 1.4 qcm Grösse mit genügendem. Unterhautgewebe ẉurden sorgfältig mit scharfem Skalpell herausgeschnitten, in Zelloidin eingebettet und in Flächenschnittserien zerlegt. Die Schnittdicke beträgt $40 \mu$ Dicke, mit Ausnahme von $30 \mu$ der Stirnhaut. Als Färbung wurden das Hämatoxylin (Hansen)- Eosin und die Karminsäure-Phosphormolybdänsäurelösung nach Okajima angewandt. Ich habe folgende 11 Körperteile untersucht.

I. Kopf :

1. Scheitel

2. Stirn (Behaarungsgrenze)

II. Rumpf :

3. Rücken (linke Seite)

4. Brust (linke Seite) .

5. Gesäss (linke Seite)

III. Obere Extremität :

6. Oberarm (Streckseite der mittleren Höhe, links)

7. Vorderarm (Streckseite der mittleren Höhe, links)

8. Vorderarm (Beugeseite der mittleren Höhe, links)

IV. Untere Extremität :

9. Oberschenkel (Lateralseite der oberen Höhe, links)

10. Unterschenkel (Streckseite der mittleren Höhe, links)

11. Unterschenkel (Beugeseite der mittleren Höhe, links)

Die Haarwurzel, die Muskelansatzstelle und die Kontur der geschnittenen Epidermis in allen Schnitten wurden 50 mal vergrössert auf einen Papierbogen aufeinander projiziert gezeichnet, wobei es unbedingt notwendig ist, als Merkmale die tadellos angebrachten Richtzeichen anzubringen.

Ich gebrauchte folgende Gleichungen nach Okajima (9), um den Winkel, die Länge etc. zu berechnen : 
1. Haut-Haarwurzel-Winkel ( $\delta$-Winkel) (Fig. 1):

$$
\delta=90-\delta^{\prime}
$$

$\cos \delta^{\prime}=\cos \mathrm{y} \cos \psi+\sin \mathrm{y} \sin \psi \cos \alpha$

$\psi=\Varangle O \mathrm{Opp}^{\prime}=\tan ^{-1} \frac{\mathrm{d}}{\mathrm{e}}$

$$
\mathrm{y}=\Varangle \mathrm{OQQ}^{\prime}=\tan ^{-1} \frac{\mathrm{d}^{\prime}}{\mathrm{e}^{\prime}}
$$

$\alpha=$ auf Projektionsbild direkt gemessen; Abweichungswinkel der Neigungsrichtung $\left(\mathrm{OQ}^{\prime}\right)$ der Ebene (Hautfläche F) von der Projektion $\left(\mathrm{OP}^{\prime}\right)$ der Haarwurzel (OP).

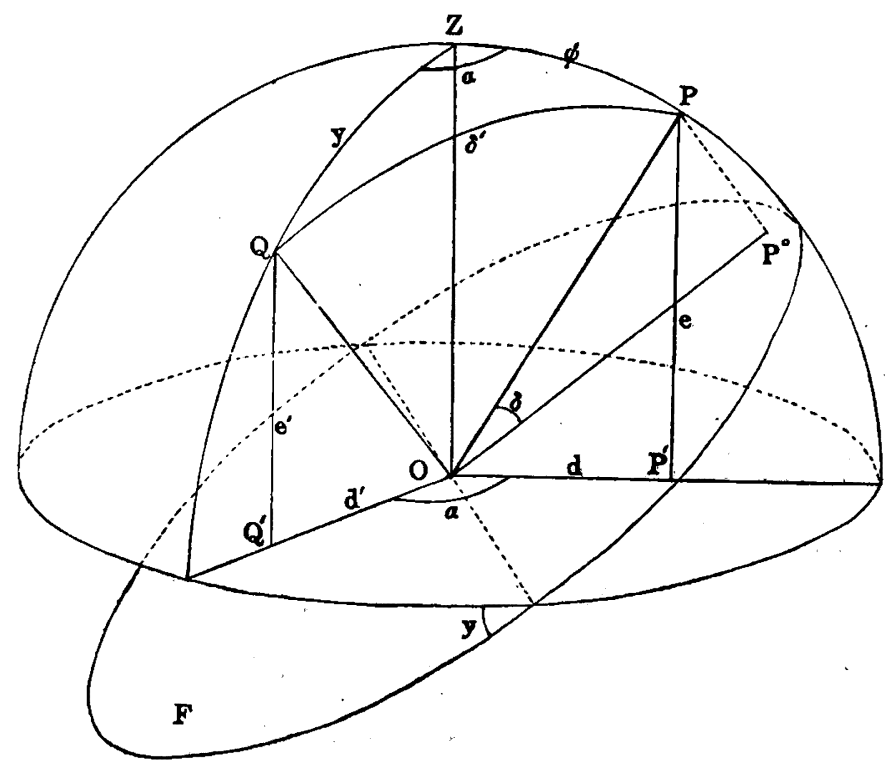

Fig. 1.

$\mathrm{d}, \mathrm{d}^{\prime}=$ auf Projektionsbild direkt gemessen.

$\mathrm{e}, \mathbf{e}^{\prime}=$ Schnittzahl $\times$-dicke $\times$ Vergrösserung.

Über die Bestimmung der Neigungsrichtung und des -winkels $(y)$ der Hautfläche siehe die Arbeit von Okajima (9).

Zur Berechnung der Haarwurzellänge und Ansatzhöhe des Arrektors

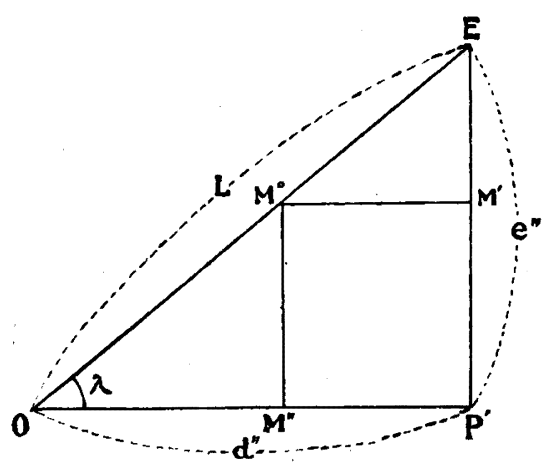

Fig. 2. 
am Haarbalg ist es nicbt nötig, auf die schräge Schnittführung besonders einzugeben (Fig. 2) :

2. Haarwurzellänge $(\mathrm{L})=\frac{\sqrt{\mathrm{e}^{1 / 2}+\mathrm{d}^{\prime \prime 2}}}{\text { Vergrösserung }}=\frac{\mathrm{e}^{\prime \prime}}{\sin \lambda \times \text { Vergrösseruug }}$

oder $\frac{d^{\prime \prime}}{\cos \lambda \times \text { Vergrösserung }}$

$\mathrm{d}^{\prime \prime}=$ Projizierte Länge des Haars,

$e^{\prime \prime}=$ Projektionstiefe von der Epidermis zur Haarpapille,

$\lambda=$ Nicht korrigierter Haut-Haarwurzel-Winkel.

3. Länge von Ansatzstelle bis zur Haarpapille $=\frac{\mathbf{M}^{\circ} \mathbf{M}^{\prime \prime}}{\sin \lambda \times \text { Vergrösserung }}$

4. Länge vom Epithel bis zur Ansatzstelle=Haarwurzellänge-Länge von Ansatzstelle bis zur Haarpapille

5. Die vertikale Tiefe vom Epithel bis zur Haarpapille (EP) :

$\mathrm{EP}=$ Haarwurzellänge $\times \sin \delta$

6. Die vertikale Tiefe vom Epithel bis zur Muskelansatzstelle am Haarbalg (EM) :

$\mathrm{EM}=$ Länge vom Epithel bis zur Ansatzstelle $\times \sin \delta$.

Im folgenden werde ich nur einige Beispiele (Haarnummer $g^{\prime}$ am Scheitel) von Berechnungen aus den zahlreichen Untersuchungsfällen nach den obigen Gleichungen angeben (s. Okajima u. Koibuchi (8)).

1. Haut-Haarwurzel-Winkel $(\boldsymbol{\delta})$ :

$\mathrm{Da} \alpha=105^{\circ} 41^{\prime}$

$\mathrm{d}=16.5 \mathrm{~mm}$

$\mathrm{e}=8$ Schnitte $\times 0.04 \mathrm{~mm} \times 50 \mathrm{mal}=16 \mathrm{~mm}$

$y=\tan ^{-1} \frac{0.04 \times 4 \times 50}{40}=\frac{8}{40}=0.2$

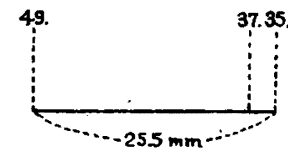

Fig. 3.

$\phi=\tan ^{-1} \frac{16.5}{16}=1.031$

und $\Varangle \delta=90^{\circ}-\delta^{\prime}$

$\cos \delta^{\prime}=\cos \psi \cos \mathrm{y}+\sin \psi \sin \mathrm{y} \cos \alpha \quad$ ist

so $\cos \delta^{\prime}=0.6963 \times 0.9806+0.7177 \times 0.1962 \times-0.2703=0.6448$

$\Varangle \delta^{\prime \prime}=49^{\circ} 51^{\prime} \quad \therefore \chi^{\delta}=90^{\circ}-49^{\circ} 51^{\prime}=40^{\circ} 9^{\prime}$.

2. Haarwurzellänge $=\frac{\mathrm{e}^{\prime \prime}}{\sin \lambda \times \text { Vergrösserung }}=\frac{14 \times 0.04 \times 50}{0.6961 \times 50}=0.804 \mathrm{~mm}$ 


$$
\left(\lambda=\tan ^{-1} \frac{\mathrm{e}}{\mathrm{d}}=\tan ^{-1} \frac{16}{16.5}=0.9697 \quad \therefore \lambda=44^{\circ} 7^{\prime}\right)
$$

3. Länge von Ansatzstelle bis zur Haarpapille $=\frac{\mathrm{M}^{\circ} \mathrm{M}^{\prime \prime}}{\sin \lambda \times \text { Vergrösserung }}$

$$
=\frac{2 \times 0.04 \times 50}{0.6961 \times 50}=0.115 \mathrm{~mm}
$$

4. Länge vom Epithel bis zur Ansatzstelle $=0.804-0.115=0.689 \mathrm{~mm}$

5. Die vertikale Tiefe vom Epithel bis zur Haarpapille (EP) $=0.804$ $\times 0.64478=0.518 \mathrm{~mm}$

6. Die vertikale Tiefe vom Epithel bis zur Ansatzstelle $(E M)=0.689$ $\times 0.64478=0.444 \mathrm{~mm}$

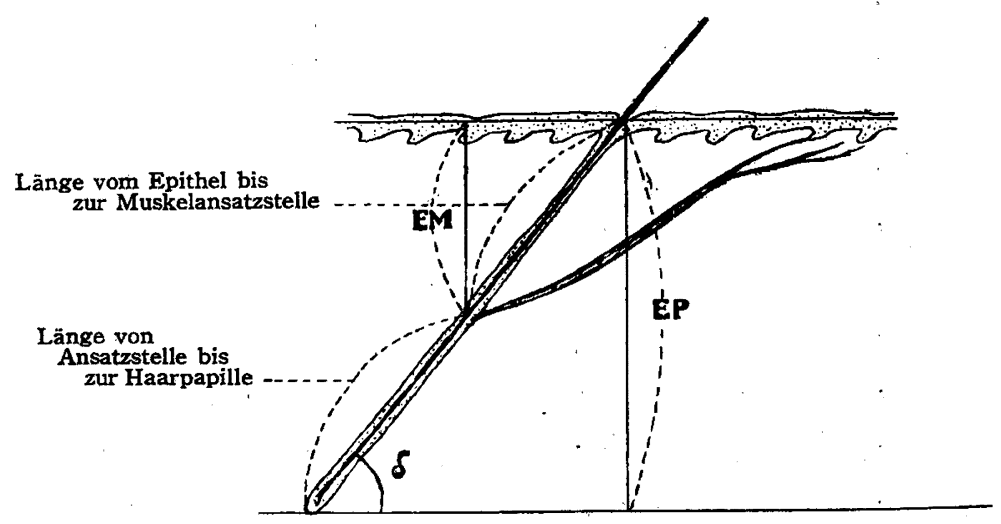

Fig. 4.

\section{Eigene Befunde und Zusammenstellung.}

Der Haut-Haarwurzel-Winkel $(\boldsymbol{\delta})$, die Haarwurzellänge, die Muskelansatzhöhe am Haarbalg und die vertikale Tiefe vom Epithel bis zur Haarpapille (EP) oder bis zur Muskelansatzstelle (EM) in verschiedenen Körperteilen, welche ich nach den obigen Methoden berechnet habe, sind in folgenden Tabellen zusammengestellt. Aber hier ist der nicht korrigierte Wert des Haut-Haarwurzel-Winkels $(\lambda)$ dargestellt, also muss man diesen Werten den Fehler $( \pm \varepsilon)$, welcher wegen der schrägen Schnittführung bedingt wird, aber meistens nur klein ist, hinzufügen, so $\delta=\lambda \pm \varepsilon$. Die vertikale Tiefe vom Epithel bis zur Haarpapille oder bis zur Muskelansatzstelle ist auch nicht korrigiert angegeben (EP' und EM'), da wegen der schrägen Schnittführung auch hier ein geringer Fehler sich eingeschlichen hat. 
Tabelle 1. Scheitel.

\begin{tabular}{|c|c|c|c|c|c|c|c|}
\hline \multirow{2}{*}{\multicolumn{2}{|c|}{$\begin{array}{c}\text { Haar- } \\
\text { nummer }\end{array}$}} & \multirow{2}{*}{ 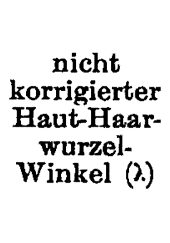 } & \multirow{2}{*}{$\begin{array}{c}\text { Haar- } \\
\text { wurzel- } \\
\text { länge }\end{array}$} & \multicolumn{2}{|c|}{$\begin{array}{l}\text { Ansatzhöhe des Arrek. } \\
\text { tors an Haarwurzel }\end{array}$} & \multirow[b]{2}{*}{$\mathbf{E P}^{\prime}$} & \multirow[b]{2}{*}{ EM' } \\
\hline & & & & $\begin{array}{l}\text { Iänge vom } \\
\text { Epithel bis } \\
\text { zur Ansatz. } \\
\text { stelle }\end{array}$ & $\begin{array}{c}\text { Iänge von } \\
\text { Ansatzstelle } \\
\text { bis zur } \\
\text { Haarpapille }\end{array}$ & & \\
\hline \multicolumn{2}{|l|}{$\mathbf{a}$} & $26^{\circ} 34^{\prime}$ & $2.321 \mathrm{~mm}$ & $0.890 \mathrm{~mm}$ & $1.431 \mathrm{~mm}$ & $1.038 \mathrm{~mm}$ & $0.398 \mathrm{~mm}$ \\
\hline \multicolumn{2}{|l|}{ b } & $26^{\circ} 56^{\prime}$ & 2.124 & 0.799 & 1.325 & 0.962 & 0.362 \\
\hline \multicolumn{2}{|l|}{ c } & $29^{\circ} \quad 3^{\prime}$ & 2.058 & 0.822 & 1.236 & 0.999 & 0.399 \\
\hline \multicolumn{2}{|l|}{ d } & $29^{\circ} 29^{\prime}$ & 2.195 & 0.895 & 1.300 & 1.080 & 0.440 \\
\hline \multicolumn{2}{|l|}{$\mathrm{e}$} & $30^{\circ} \quad 6^{\prime}$ & 0.797 & 0.717 & 0.080 & 0.370 & 0.360 \\
\hline \multicolumn{2}{|l|}{ f } & $30^{\circ} 25^{\prime}$ & 2.134 & 0.870 & 1.264 & 1.080 & 0.440 \\
\hline \multicolumn{2}{|l|}{$g$} & $31^{\circ} 24^{\prime}$ & 2.222 & 0.917 & 1.305 & 1.157 & 0.478 \\
\hline \multicolumn{2}{|l|}{$g^{\prime}$} & $44^{\circ} \quad 7^{\prime}$ & 0.804 & 0.689 & 0.115 & 0.560 & 0.480 \\
\hline \multicolumn{2}{|l|}{$\mathbf{h}$} & $32^{\circ} 44^{\prime}$ & 2.000 & 0.816 & 1.184 & 1.082 & 0.441 \\
\hline \multicolumn{2}{|l|}{$\mathrm{i}$} & $33^{\circ} \quad 8^{\prime}$ & 2.271 & 0.881 & 1.390 & 1.241 & 0.482 \\
\hline \multicolumn{2}{|l|}{$j$} & $33^{\circ} 41^{\prime}$ & 2.166 & 0.796 & 1.370 & 1.202 & 0.441 \\
\hline \multicolumn{2}{|l|}{$\mathbf{k}$} & $35^{\circ} 39^{\prime}$ & 0.825 & 0.688 & 0.137 & 0.481 & 0.401 \\
\hline \multicolumn{2}{|l|}{1} & $39^{\circ} 14^{\prime}$ & 0.632 & 0.632 & 0 & 0.400 & 0.400 \\
\hline \multirow{2}{*}{$\begin{array}{l}\text { Durch- } \\
\text { schnitt }\end{array}$} & $\mathbf{H}$ & $31^{\circ} 32^{\prime}$ & $\begin{array}{l}2.166 \text { (lang) } \\
0.751 \text { (kurz) }\end{array}$ & & & 0.924 & 0.420 \\
\hline & $\mathbf{N}$ & $44^{\circ} \quad 7^{\prime}$ & 0.804 & & & 0.560 & 0.480 \\
\hline
\end{tabular}

Absoluter Haut-Haarwurzel-Winkel $=\lambda_{1} \pm \varepsilon_{1}, \lambda_{2} \pm \varepsilon_{2}$

$\mathbf{E P}^{\prime}=$ Die scheinbare vertikale Tiefe vom Epithel bis zur Haarpapille,

$\mathbf{E M}^{\prime}=$ Die scheinbare vertikale Tiefe vom Epithel bis zur Muskelansatzstelle,

$\mathrm{H}=$ Haupthar,

$\mathrm{N}$ und' = Nebenhar. 
Tabelle 2. Stirn.

\begin{tabular}{|c|c|c|c|c|c|c|c|}
\hline \multirow{2}{*}{\multicolumn{2}{|c|}{$\underset{\text { nummer }}{\text { Haar- }}$}} & \multirow{2}{*}{$\begin{array}{c}\text { nicht } \\
\text { korrigierter } \\
\text { Haut-Haar- } \\
\text { wurzel- } \\
\text { Winkel ( }) \text { (.) }\end{array}$} & \multirow{2}{*}{$\begin{array}{l}\text { Haar- } \\
\text { wurzel- } \\
\text { länge }\end{array}$} & \multicolumn{2}{|c|}{$\begin{array}{l}\text { Ansatzhöhe des Arrek- } \\
\text { tors an Haarwurzel }\end{array}$} & \multirow[b]{2}{*}{$\mathbf{E P}$} & \multirow[b]{2}{*}{$\mathbf{E M}^{\prime}$} \\
\hline & & & & $\begin{array}{c}\text { Jänge vom } \\
\text { Epithel bis } \\
\text { zur Ansatz- } \\
\text { stelle }\end{array}$ & $\begin{array}{l}\text { Jänge von } \\
\text { Ansatzstelle } \\
\text { bis zur } \\
\text { Haarpapille }\end{array}$ & & \\
\hline \multicolumn{2}{|l|}{ a } & $28^{\circ} 36^{\prime}$ & $1.569 \mathrm{~mm}$ & $1.005 \mathrm{~mm}$ & $0.564 \mathrm{~mm}$ & $0.751 \mathrm{~mm}$ & $0-481 \mathrm{~mm}$ \\
\hline \multicolumn{2}{|l|}{ b } & $30^{\circ} 58^{\prime}$ & 0.700 & 0.642 & 0.058 & 0.360 & 0.330 \\
\hline \multicolumn{2}{|l|}{ c } & $31^{\circ} 14^{\prime}$ & 1.100 & 0.811 & 0.289 & 0.570 & 0.421 \\
\hline \multicolumn{2}{|l|}{$d$} & $31^{\circ} 32^{\prime}$ & 0.515 & 0.400 & 0.115 & 0.269 & 0.209 \\
\hline \multicolumn{2}{|l|}{ e } & $31^{\circ} 39^{\prime}$ & 1.143 & 0.743 & 0.400 & 0.600 & 0.390 \\
\hline \multicolumn{2}{|l|}{$\mathbf{f}$} & $32^{\circ} 33^{\prime}$ & 1.059 & 0.669 & 0.390 & 0.570 & 0.360 \\
\hline \multicolumn{2}{|l|}{ g } & $34^{\circ} \quad 3^{\prime}$ & 1.339 & 0.589 & 0.750 & 0.750 & 0.330 \\
\hline \multicolumn{2}{|l|}{$\mathbf{h}$} & $35^{\circ} 13^{\prime}$ & 0.676 & 0.572 & 0.104 & 0.390 & 0.330 \\
\hline \multicolumn{2}{|l|}{ i } & $35^{\circ} 13^{\prime}$ & 0.833 & 0.833 & 0 & 0.480 & 0.480 \\
\hline \multicolumn{2}{|l|}{$i^{\prime}$} & $44^{\circ} 17^{\prime}$ & 0.559 & - & - & 0.340 & - \\
\hline \multicolumn{2}{|l|}{$\mathbf{j}$} & $36^{\circ} 34^{\prime}$ & 1.107 & 0.755 & 0.352 & 0.660 & 0.450 \\
\hline \multirow{2}{*}{$\begin{array}{l}\text { Durch- } \\
\text { schnitt }\end{array}$} & $\mathbf{H}$ & $32^{\circ} 46^{\prime}$ & $\begin{array}{l}1.004 \text { (lang) } \\
0.681 \text { (kuxz) }\end{array}$ & & & 0.540 & 0.378 \\
\hline & $\mathbf{N}$ & $44^{\circ} 17^{\prime}$ & 0.559 & & & 0.340 & - \\
\hline
\end{tabular}

Tabelle 3. Rücken.

\begin{tabular}{|c|c|c|c|c|c|c|c|}
\hline \multirow{2}{*}{\multicolumn{2}{|c|}{$\underset{\text { nummer }}{\text { Haar- }}$}} & \multirow{2}{*}{$\begin{array}{c}\text { nicht } \\
\text { korrigierter } \\
\text { Haut-Haar. } \\
\text { wurzel- } \\
\text { Winkel ( ..) }\end{array}$} & \multirow{2}{*}{$\begin{array}{c}\text { Haar- } \\
\text { wurzel- } \\
\text { länge }\end{array}$} & \multicolumn{2}{|c|}{$\begin{array}{l}\text { Ansatzhöhe des Arrek- } \\
\text { tors an Haarwurzel }\end{array}$} & \multirow[b]{2}{*}{$\mathbf{E P}^{\prime}$} & \multirow[b]{2}{*}{$\mathbf{E} \mathbf{M}^{\prime}$} \\
\hline & & & & $\begin{array}{c}\text { Iänge vom } \\
\text { Epithel bis } \\
\text { zur Ansatz- } \\
\text { stelle }\end{array}$ & $\begin{array}{l}\text { Iänge von } \\
\text { Ansatzstelle } \\
\text { bis zur } \\
\text { Haarpapille }\end{array}$ & & \\
\hline \multicolumn{2}{|l|}{$\mathbf{a}$} & $33^{\circ} 42^{\prime}$ & $0.722 \mathrm{~mm}$ & $0.434 \mathrm{~mm}$ & $0.288 \mathrm{~mm}$ & $0.401 \mathrm{~mm}$ & $0.241 \mathrm{~mm}$ \\
\hline \multicolumn{2}{|l|}{$a^{\prime}$} & $32^{\circ} 37^{\prime}$ & 0.592 & 一 & - & 0.320 & - \\
\hline \multicolumn{2}{|l|}{ b } & $36^{\circ} 15^{\prime}$ & 0.743 & 0.540 & 0.203 & 0.439 & 0.319 \\
\hline \multicolumn{2}{|l|}{ c } & $38^{\circ} \quad 3^{\prime}$ & 0.584 & - & - & 0.360 & - \\
\hline \multicolumn{2}{|l|}{$\mathrm{c}^{\prime}$} & $52^{\circ} \quad 7^{\prime}$ & 0.456 & - & - & 0.360 & - \\
\hline \multicolumn{2}{|l|}{ d } & $38^{\circ} \quad 6^{\prime}$ & 0.647 & 0.582 & 0.065 & 0.399 & 0.359 \\
\hline \multicolumn{2}{|l|}{$d^{\prime}$} & $39^{\circ} 17^{\prime}$ & 0.570 & - & - & 0.361 & - \\
\hline \multicolumn{2}{|l|}{ e } & $39^{\circ} 48^{\prime}$ & 0.625 & 0.563 & 0.062 & 0.400 & 0.360 \\
\hline \multicolumn{2}{|l|}{$\mathbf{f}$} & $40^{\circ} \cdot 36^{\prime}$ & 0.554 & 0.493 & 0.061 & 0.361 & 0.321 \\
\hline \multicolumn{2}{|l|}{$f^{\prime}$} & $42^{\circ} 43^{\prime}$ & 0.531 & - & - & 0.360 & - \\
\hline \multicolumn{2}{|l|}{ g } & $41^{\circ} 59^{\prime}$ & 0.539 & 0.419 & 0.120 & 0.361 & 0.280 \\
\hline \multirow{2}{*}{$\begin{array}{l}\text { Durch- } \\
\text { schnitt }\end{array}$} & $\mathbf{H}$ & $38^{\circ} 21^{\prime}$ & 0.631 & & 0.389 & 0.313 \\
\hline & $\mathbf{N}$ & $41^{\circ} 41^{\prime}$ & 0.538 & & & 0.350 & - \\
\hline
\end{tabular}


Tabelle 4. Brust.

\begin{tabular}{|c|c|c|c|c|c|c|c|}
\hline \multirow{2}{*}{\multicolumn{2}{|c|}{$\begin{array}{l}\text { Haar- } \\
\text { nummer }\end{array}$}} & \multirow{2}{*}{$\begin{array}{c}\text { nicht } \\
\text { korrigierter } \\
\text { Haut-Haar- } \\
\text { wurzel- } \\
\text { Winkel ( (.) }\end{array}$} & \multirow{2}{*}{$\begin{array}{c}\text { Haar- } \\
\text { wurzel- } \\
\text { länge }\end{array}$} & \multicolumn{2}{|c|}{$\begin{array}{l}\text { Ansatzhöhe des Arrek- } \\
\text { tors an Haarwurzel }\end{array}$} & \multirow[b]{2}{*}{$\mathbf{E P}^{\prime}$} & \multirow[b]{2}{*}{$\mathbf{E M}^{\prime}$} \\
\hline & & & & $\begin{array}{c}\text { Iange vom } \\
\text { Epithel bis } \\
\text { zur Ansatz- } \\
\text { stelle }\end{array}$ & $\begin{array}{c}\text { Sänge von } \\
\text { Ansatzstelle } \\
\text { bis zur } \\
\text { Haarpapille }\end{array}$ & & \\
\hline \multicolumn{2}{|l|}{ a } & $32^{\circ} 42^{\prime}$ & $0.518 \mathrm{~mm}$ & $0.444 \mathrm{~mm}$ & $0.074 \mathrm{~mm}$ & $0.280 \mathrm{~mm}$ & $0.240 \mathrm{~mm}$ \\
\hline \multicolumn{2}{|l|}{$\mathbf{a}^{\prime}$} & $38^{\circ} 40^{\prime}$ & 0.449 & 0.385 & 0.064 & 0.281 & 0.241 \\
\hline \multicolumn{2}{|l|}{ b } & $34^{\circ} 42^{\prime}$ & 0.632 & 0.491 & 0.141 & 0.360 & 0.280 \\
\hline \multicolumn{2}{|l|}{$b^{\prime}$} & $43^{\circ} 27^{\prime}$ & 0.523 & 一 & - & 0.360 & - \\
\hline \multicolumn{2}{|l|}{ c } & $36^{\circ} \quad 2^{\prime}$ & 0.816 & 0.680 & 0.136 & 0.480 & 0.400 \\
\hline \multicolumn{2}{|l|}{$\mathrm{c}^{\prime}$} & $41^{\circ} 38^{\prime}$ & 0.723 & - & - & 0.480 & - \\
\hline \multicolumn{2}{|l|}{ d } & $36^{\circ} 32^{\prime}$ & 0.671 & 0.469 & 0.202 & 0.399 & 0.279 \\
\hline \multicolumn{2}{|l|}{$\mathrm{d}^{\prime}$} & $42^{\circ} 43^{\prime}$ & 0.531 & - & - & 0.360 & - \\
\hline \multicolumn{2}{|l|}{ e } & $38^{\circ} \quad 3^{\prime}$ & 0.584 & 0.454 & 0.130 & 0.360 & 0.280 \\
\hline \multicolumn{2}{|l|}{$\mathrm{e}^{\prime}$} & $43^{\circ} 27^{\prime}$ & 0.523 & - & - & 0.360 & - \\
\hline \multicolumn{2}{|l|}{ f } & $40^{\circ} \quad 47^{\prime}$ & 0.673 & 0.551 & 0.122 & 0.440 & 0.360 \\
\hline \multicolumn{2}{|l|}{$f^{\prime}$} & $45^{\circ}$ & 0.395 & - & - & 0.279 & - \\
\hline \multicolumn{2}{|l|}{ g } & $41^{\circ} 53^{\prime}$ & 0.778 & 0.598 & 0.180 & 0.519 & 0.399 \\
\hline \multicolumn{2}{|l|}{$\mathrm{g}^{\prime}$} & $50^{\circ} 29^{\prime}$ & 0.622 & - & - & 0.480 & - \\
\hline \multicolumn{2}{|l|}{$g^{\prime \prime}$} & $39^{\circ} 10^{\prime}$ & 0.696 & - & - & 0.440 & - \\
\hline \multicolumn{2}{|l|}{$\mathbf{h}$} & $42^{\circ} 10^{\prime}$ & 0.714 & 0.595 & 0.119 & 0.479 & 0.399 \\
\hline \multicolumn{2}{|l|}{$\mathrm{h}^{\prime}$} & $44^{\circ} 13^{\prime}$ & 0.688 & - & - & 0.480 & - \\
\hline \multirow{2}{*}{$\begin{array}{l}\text { Durch- } \\
\text { schnitt }\end{array}$} & $\mathbf{H}$ & $37^{\circ} 51^{\prime}$ & 0.673 & & & 0.415 & 0.330 \\
\hline & $\mathrm{N}$ & $43^{\circ} 12^{\prime}$ & 0.572 & & & 0.391 & 0.241 \\
\hline
\end{tabular}


Tabelle 5. Gesäss.

\begin{tabular}{|c|c|c|c|c|c|c|c|}
\hline \multirow{2}{*}{$\begin{array}{l}\text { Haar- } \\
\text { nummer }\end{array}$} & & \multirow{2}{*}{$\begin{array}{c}\text { nicht } \\
\text { korrigierter } \\
\text { Haut-Haar- } \\
\text { wurzel- } \\
\text { Winkel ( ( .) }\end{array}$} & \multirow{2}{*}{$\begin{array}{l}\text { Haar- } \\
\text { wurzel- } \\
\text { länge }\end{array}$} & \multicolumn{2}{|c|}{$\begin{array}{l}\text { Ansatzhöhe des Arrek- } \\
\text { tors an Haarwurzel }\end{array}$} & \multirow[b]{2}{*}{$\mathbf{E P}^{\prime}$} & \multirow[b]{2}{*}{$\mathrm{EM}^{\prime}$} \\
\hline & & & & $\begin{array}{c}\text { Länge vom } \\
\text { Epithel bis } \\
\text { zur Ansatz- } \\
\text { stelle }\end{array}$ & $\begin{array}{c}\text { Länge von } \\
\text { Ansatzstelle } \\
\text { bis zur } \\
\text { Haarpapille } \\
\end{array}$ & & \\
\hline \multicolumn{2}{|l|}{$\mathbf{a}$} & $34^{\circ} 31^{\prime}$ & $0.919 \mathrm{~mm}$ & $0.778 \mathrm{~mm}$ & $0.141 \mathrm{~mm}$ & $0.521 \mathrm{~mm}$ & $0.441 \mathrm{~mm}$ \\
\hline \multicolumn{2}{|l|}{$\mathbf{a}^{\prime}$} & $51^{\circ} 44^{\prime}$ & 0.662 & - & $-{ }^{\prime}$ & 0.520 & - \\
\hline \multicolumn{2}{|l|}{ b } & $35^{\circ} 48^{\prime}$ & 0.753 & 0.616 & 0.137 & 0.440 & 0.360 \\
\hline \multicolumn{2}{|l|}{$b^{\prime}$} & $38^{\circ} 39^{\prime}$ & 0.641 & - & - & 0.400 & - \\
\hline \multicolumn{2}{|l|}{ c } & $37^{\circ} 11^{\prime}$ & 0.728 & 0.662 & 0.066 & 0.440 & 0.400 \\
\hline \multicolumn{2}{|l|}{$\mathrm{c}^{\prime}$} & $37^{\circ} 40^{\prime}$ & 0.719 & - & - & 0.439 & - \\
\hline \multicolumn{2}{|l|}{ d } & $37^{\circ} 45^{\prime}$ & 0.784 & 0.588 & 0.196 & 0.480 & 0.360 \\
\hline \multicolumn{2}{|l|}{$d^{\prime}$} & $38^{\circ} 40^{\prime}$ & 0.705 & 0.577 & 0.128 & 0.440 & 0.361 \\
\hline \multicolumn{2}{|l|}{$\mathrm{e}$} & $39^{\circ} \quad 6^{\prime}$ & 0.825 & 0.571 & 0.254 & 0.520 & $0 . \dot{3} 60$ \\
\hline \multicolumn{2}{|l|}{$e^{\prime}$} & $40^{\circ} 27^{\prime}$ & 0.802 & 一 & $-{ }^{\prime}$ & 0.520 & 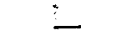 \\
\hline \multicolumn{2}{|l|}{ f } & $39^{\circ} \quad 8^{\prime}$ & 0.759 & 0.505 & 0.254 & 0.479 & 0.319 \\
\hline \multicolumn{2}{|l|}{$f^{\prime}$} & $45^{\circ} 35^{\prime}$ & 0.672 & - & - & 0.480 & - \\
\hline \multicolumn{2}{|l|}{ g } & $39^{\circ} 22^{\prime}$ & 0.820 & 0.568 & 0.252 & 0.520 & 0.360 \\
\hline \multicolumn{2}{|l|}{$\mathrm{g}^{\prime}$} & $39^{\circ} \quad 8^{\prime}$ & 0.759 & 一 & - & 0.479 & - \\
\hline \multicolumn{2}{|l|}{ h } & $39^{\circ} 32^{\prime}$ & 0.818 & 0.629 & 0.189 & 0.521 & 0.400 \\
\hline \multicolumn{2}{|l|}{ i } & $40^{\circ} 36^{\prime}$ & 0.738 & 0.492 & 0.246 & 0.480 & 0.320 \\
\hline \multicolumn{2}{|l|}{$i^{\prime}$} & $45^{\circ} \quad 2^{\prime}$ & 0.678 & 0.452 & 0.226 & 0.480 & 0.320 \\
\hline \multicolumn{2}{|l|}{$\mathrm{i}^{\prime \prime}$} & $46^{\circ} 12^{\prime}$ & 0.665 & 0.443 & 0.222 & 0.480 & 0.320 \\
\hline \multicolumn{2}{|l|}{$\mathrm{j}$} & $41^{\circ} 21^{\prime}$ & 0.667 & 0.606 & 0.061 & 0.441 & 0.400 \\
\hline \multicolumn{2}{|l|}{$j^{\prime}$} & $49^{\circ} 56^{\prime}$ & 0.574 & 一 & $-{ }^{\prime}$ & 0.439 & - \\
\hline \multicolumn{2}{|l|}{$j^{\prime \prime}$} & $43^{\circ} 7^{\prime}$ & 0.643 & - & - & 0.439 & - \\
\hline \multirow{2}{*}{$\begin{array}{l}\text { Durch- } \\
\text { schnitt }\end{array}$} & $\mathrm{H}$ & $38^{\circ} 26^{\prime}$ & 0.781 & & & 0.484 & 0.372 \\
\hline & $\mathbf{N}$ & $43^{\circ} 17^{\prime}$ & 0.684 & & & 0.465 & 0.334 \\
\hline
\end{tabular}


Tabelle 6. Oberarm (Streckseite).

\begin{tabular}{|c|c|c|c|c|c|c|c|}
\hline \multirow{2}{*}{\multicolumn{2}{|c|}{$\begin{array}{c}\text { Haar- } \\
\text { nummer }\end{array}$}} & \multirow{2}{*}{$\begin{array}{c}\text { nicht } \\
\text { korrigierter } \\
\text { Haut-Haar- } \\
\text { wurzel- } \\
\text { Winkel }(\lambda)\end{array}$} & \multirow{2}{*}{$\begin{array}{c}\text { Haar- } \\
\text { wurzel- } \\
\text { länge }\end{array}$} & \multicolumn{2}{|c|}{$\begin{array}{l}\text { Ansatzhöhe des Arrek- } \\
\text { tors an Haarwurzel }\end{array}$} & \multirow[b]{2}{*}{ EP' } & \multirow[b]{2}{*}{$\mathrm{EM}^{\prime}$} \\
\hline & & & & $\begin{array}{l}\text { Iänge vom } \\
\text { Epithel bis } \\
\text { zur Ansatz } \\
\text { stelle }\end{array}$ & $\begin{array}{c}\text { Iänge von } \\
\text { Ansatzstelle } \\
\text { bis zur } \\
\text { Haarpapille }\end{array}$ & & \\
\hline \multicolumn{2}{|l|}{$\mathbf{a}$} & $43^{\circ} 16^{\prime}$ & $0.467 \mathrm{~mm}$ & $0.409 \mathrm{~mm}$ & $0.058 \mathrm{~mm}$ & $0.320 \mathrm{~mm}$ & $0.280 \mathrm{~mm}$ \\
\hline \multicolumn{2}{|l|}{$a^{\prime}$} & $47^{\circ} 13^{\prime}$ & 0.436 & - & - & 0.320 & - \\
\hline \multicolumn{2}{|l|}{ b } & $45^{\circ} 53^{\prime}$ & 0.446 & 0.279 & 0.167 & 0.320 & 0.200 \\
\hline \multicolumn{2}{|l|}{ c } & $46^{\circ} 20^{\prime}$ & 0.608 & 0.497 & 0.111 & 0.440 & 0.360 \\
\hline \multicolumn{2}{|l|}{$\mathrm{d}$} & $48^{\circ}$ & 0.538 & 0.430 & 0.108 & 0.400 & 0.320 \\
\hline \multicolumn{2}{|l|}{$\mathrm{e}$} & $48^{\circ}$ & 0.538 & 0.430 & 0.108 & 0.400 & 0.320 \\
\hline \multicolumn{2}{|l|}{ f } & $48^{\circ} 14^{\prime}$ & 0.536 & 0.482 & 0.054 & 0.400 & 0.360 \\
\hline \multicolumn{2}{|l|}{$\mathrm{g}$} & $48^{\circ} 34^{\prime}$ & 0.640 & 0.373 & 0.267 & 0.480 & 0.280 \\
\hline \multicolumn{2}{|l|}{$\mathrm{g}^{\prime}$} & $56^{\circ} 19^{\prime}$ & 0.577 & - & - & 0.480 & - \\
\hline \multicolumn{2}{|l|}{ h } & $49^{\circ} 11^{\prime}$ & 0.582 & 0.476 & 0.106 & 0.440 & 0.360 \\
\hline \multicolumn{2}{|l|}{ i } & $49^{\circ} 56^{\prime}$ & 0.575 & 0.364 & 0.157 & 0.440 & 0.279 \\
\hline \multicolumn{2}{|l|}{$\mathrm{i}^{\prime}$} & $58^{\circ} 28^{\prime}$ & 0.516 & - & - & 0.440 & - \\
\hline \multicolumn{2}{|l|}{$\mathrm{i}^{\prime \prime}$} & $51^{\circ} 20^{\prime}$ & 0.512 & - & - & 0.400 & - \\
\hline \multicolumn{2}{|l|}{$\mathrm{j}$} & $49^{\circ} 57^{\prime}$ & 0.523 & 0.418 & 0.105 & 0.400 & 0.320 \\
\hline \multicolumn{2}{|l|}{$\mathbf{k}$} & $50^{\circ} 11^{\prime}$ & 0.625 & 0.469 & 0.156 & 0.480 & 0.360 \\
\hline \multicolumn{2}{|l|}{$\mathrm{k}^{\prime}$} & $65^{\circ} 34^{\prime}$ & 0.484 & - & - & 0.441 & - \\
\hline \multicolumn{2}{|l|}{$\mathrm{k}^{\prime \prime}$} & $58^{\circ} 52^{\prime}$ & 0.561 & 0.421 & 0.140 & 0.480 & 0.360 \\
\hline \multicolumn{2}{|l|}{1} & $50^{\circ} 27^{\prime}$ & 0.518 & 0.414 & 0.104 & 0.399 & 0.319 \\
\hline \multicolumn{2}{|l|}{$\mathrm{m}$} & $50^{\circ} 29^{\prime}$ & 0.519 & 0.415 & 0.104 & 0.400 & 0.320 \\
\hline \multicolumn{2}{|l|}{$\mathbf{n}$} & $50^{\circ} 29^{\prime}$ & 0.518 & 0.414 & 0.104 & 0.400 & 0.319 \\
\hline \multicolumn{2}{|l|}{ o } & $55^{\circ} 30^{\prime}$ & 0.582 & 0.436 & 0.146 & 0.480 & 0.359 \\
\hline \multicolumn{2}{|l|}{$\sigma^{\prime}$} & $56^{\circ} \quad 37^{\prime}$ & 0.527 & - & - & 0.440 & - \\
\hline \multirow{2}{*}{$\begin{array}{l}\text { Durch- } \\
\text { schnitt }\end{array}$} & H & $48^{\circ} 58^{\prime}$ & 0.548 & & & 0.413 & 0.317 \\
\hline & $\mathbf{N}$ & $56^{\circ} 2 \sigma^{\circ}$ & 0.516 & & & 0.429 & 0.360 \\
\hline
\end{tabular}


Tabelle 7. Vorderarm (Streckseite).

\begin{tabular}{|c|c|c|c|c|c|c|c|}
\hline \multirow{2}{*}{\multicolumn{2}{|c|}{$\underset{\text { nummer }}{\text { Haar- }}$}} & \multirow{2}{*}{ 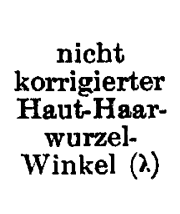 } & \multirow[b]{2}{*}{$\begin{array}{l}\text { Haar- } \\
\text { wurzel- } \\
\text { länge }\end{array}$} & \multicolumn{2}{|c|}{$\begin{array}{c}\text { Ansatzhöhe des Arrek- } \\
\text { tors an Haarwurzel }\end{array}$} & \multirow[b]{2}{*}{$\mathbf{E P}^{\prime}$} & \multirow[b]{2}{*}{$\mathbf{E M}^{\prime}$} \\
\hline & & & & $\begin{array}{l}\text { Iänge vom } \\
\text { Epithel bis } \\
\text { zur Ansatz- } \\
\text { stelle }\end{array}$ & $\begin{array}{c}\text { Länge von } \\
\text { Ansatzstelle } \\
\text { bis zur } \\
\text { Haarpapille }\end{array}$ & & \\
\hline \multicolumn{2}{|l|}{$\mathbf{a}$} & $47^{\circ} 53^{\prime}$ & $0.959 \mathrm{~mm}$ & $0.743 \mathrm{~mm}$ & $0.216 \mathrm{~mm}$ & $0.711 \mathrm{~mm}$ & $0.551 \mathrm{~mm}$ \\
\hline \multicolumn{2}{|l|}{$\mathbf{a}^{\prime}$} & $56^{\circ} 49^{\prime}$ & 0.622 & - & - & 0.521 & 一 \\
\hline \multicolumn{2}{|l|}{ b } & $48^{\circ} 49^{\prime}$ & 0.638 & 0.532 & 0.106 & 0.480 & 0.400 \\
\hline \multicolumn{2}{|l|}{ c } & $50^{\circ} 36^{\prime}$ & 0.725 & 0.570 & 0.155 & 0.560 & 0.440 \\
\hline \multicolumn{2}{|l|}{$\mathrm{d}$} & $52^{\circ} \quad 5^{\prime}$ & 0.711 & 0.610 & 0.101 & 0.561 & 0.481 \\
\hline \multicolumn{2}{|l|}{$d^{\prime}$} & $64^{\circ} 15^{\prime}$ & 0.622 & - & - & 0.560 & - \\
\hline \multicolumn{2}{|l|}{ e } & $52^{\circ} 26^{\prime}$ & 0.657 & 0.506 & 0.151 & 0.521 & 0.401 \\
\hline \multicolumn{2}{|l|}{ f } & $53^{\circ} 50^{\prime}$ & 0.644 & 0.446 & 0.198 & 0.520 & 0.360 \\
\hline \multicolumn{2}{|l|}{$g$} & $54^{\circ} 41^{\prime}$ & 0.588 & - & 一 & 0.480 & - \\
\hline \multicolumn{2}{|l|}{$g^{\prime}$} & $58^{\circ}$ & 0.566 & - & - & 0.480 & - \\
\hline \multicolumn{2}{|l|}{$g^{\prime \prime}$} & $56^{\circ} 19^{\prime}$ & 0.577 & - & - & 0.480 & - \\
\hline \multicolumn{2}{|l|}{ h } & $55^{\circ} 18^{\prime}$ & 0.633 & 0.536 & 0.097 & 0.520 & 0.441 \\
\hline \multicolumn{2}{|l|}{ i } & $60^{\circ} 15^{\prime}$ & 0.645 & - & - & 0.560 & 一 \\
\hline \multirow{2}{*}{$\begin{array}{l}\text { Durch- } \\
\text { schnitt }\end{array}$} & $\mathbf{H}$ & $52^{\circ} 53^{\prime}$ & 0.689 & & & 0.546 & 0.439 \\
\hline & $\mathbf{N}$ & $58^{\circ} 5 l^{\prime}$ & 0.597 & & & 0.510 & - \\
\hline
\end{tabular}

Tabelle 8. Vorderarm (Beugeseite).

\begin{tabular}{|c|c|c|c|c|c|c|c|}
\hline \multirow{2}{*}{\multicolumn{2}{|c|}{$\begin{array}{c}\text { Haar- } \\
\text { nummer }\end{array}$}} & \multirow{2}{*}{$\begin{array}{c}\text { nicht } \\
\text { korrigierter } \\
\text { Haut-Haar- } \\
\text { wurzel- } \\
\text { Winkel }(\gamma)\end{array}$} & \multirow{2}{*}{$\begin{array}{c}\text { Haar- } \\
\text { wurzel- } \\
\text { länge }\end{array}$} & \multicolumn{2}{|c|}{$\begin{array}{l}\text { Ansatzhöhe des Arrek- } \\
\text { tors an Haarwurzel }\end{array}$} & \multirow[b]{2}{*}{$\mathbf{E P}^{\prime}$} & \multirow[b]{2}{*}{ EM' } \\
\hline & & & & $\begin{array}{c}\text { Iänge vom } \\
\text { Epithel bis } \\
\text { zur Ansatz- } \\
\text { stelle }\end{array}$ & $\begin{array}{c}\text { Jänge von } \\
\text { Ansatzstelle } \\
\text { bis zur } \\
\text { Haarpapille }\end{array}$ & & \\
\hline \multicolumn{2}{|l|}{$\mathbf{a}$} & $32^{\circ} 19^{\prime}$ & $0.599 \mathrm{~mm}$ & $0.524 \mathrm{~mm}$ & $0.075 \mathrm{~mm}$ & $0.320 \mathrm{~mm}$ & $0.280 \mathrm{~mm}$ \\
\hline \multicolumn{2}{|l|}{ b } & $36^{\circ} 52^{\prime}$ & 0.600 & 0.533 & 0.067 & 0.360 & 0.320 \\
\hline \multicolumn{2}{|l|}{ c } & $37^{\circ} 18^{\prime}$ & 0.528 & 0.528 & 0 & 0.320 & 0.320 \\
\hline \multicolumn{2}{|l|}{ d } & $39^{\circ} \quad 8^{\prime}$ & 0.759 & 0.505 & 0.254 & 0.479 & 0.319 \\
\hline \multicolumn{2}{|l|}{$\mathbf{e}$} & $40^{\circ} \quad 2^{\prime}$ & 0.621 & 0.434 & 0.187 & 0.399 & 0.279 \\
\hline \multicolumn{2}{|l|}{ f } & $40^{\circ} 36^{\prime}$ & 0.554 & 0.431 & 0.123 & 0.361 & 0.280 \\
\hline \multicolumn{2}{|l|}{$f^{\prime}$} & $48^{\circ} 22^{\prime}$ & 0.481 & 0.374 & 0.107 & 0.359 & 0.280 \\
\hline \multicolumn{2}{|l|}{$\mathrm{g}$} & $40^{\circ} 47^{\prime}$ & 0.673 & 0.551 & 0.122 & 0.440 & 0.360 \\
\hline \multicolumn{2}{|l|}{ h } & $43^{\circ} \quad 7^{\prime}$ & 0.643 & 0.467 & 0.176 & 0.439 & 0.319 \\
\hline \multicolumn{2}{|l|}{ i } & $43^{\circ} 16^{\prime}$ & 0.466 & 0.408 & 0.058 & 0.319 & 0.280 \\
\hline \multicolumn{2}{|l|}{$\mathbf{j}$} & $43^{\circ} 45^{\prime}$ & 0.520 & 0.404 & 0.116 & 0.360 & 0.279 \\
\hline \multicolumn{2}{|l|}{$\mathbf{k}$} & $48^{\circ} 26^{\prime}$ & 0.588 & 0.428 & 0.160 & 0.440 & 0.320 \\
\hline \multirow{2}{*}{$\begin{array}{l}\text { Durch- } \\
\text { schnitt }\end{array}$} & $\mathbf{H}$ & $40^{\circ} 31^{\prime}$ & 0.596 & & & 0,385 & 0.305 \\
\hline & $\mathbf{N}$ & $48^{\circ} 22^{\prime}$ & 0.481 & & & 0.359 & 0.280 \\
\hline
\end{tabular}


Tabelle 9. Oberschenkel (Lateralseite).

\begin{tabular}{|c|c|c|c|c|c|c|c|}
\hline \multirow{2}{*}{\multicolumn{2}{|c|}{$\begin{array}{l}\text { Haar- } \\
\text { nummer }\end{array}$}} & \multirow{2}{*}{$\begin{array}{c}\text { nicht } \\
\text { korrigierter } \\
\text { Haut-Haar- } \\
\text { wurzel- } \\
\text { Winkel (ગ.) }\end{array}$} & \multirow{2}{*}{$\begin{array}{l}\text { Haar- } \\
\text { wurzel- } \\
\text { länge }\end{array}$} & \multicolumn{2}{|c|}{$\begin{array}{l}\text { Ansatzhöhe des Arrek- } \\
\text { tors an Haarwurzel }\end{array}$} & \multirow[b]{2}{*}{$\mathbf{E P}^{\prime}$} & \multirow[b]{2}{*}{$\mathrm{EM}^{\prime}$} \\
\hline & & & & $\begin{array}{l}\text { Jänge vom } \\
\text { Epithel bis } \\
\text { zur Ansatz- } \\
\text { stelle }\end{array}$ & $\begin{array}{c}\text { Länge von } \\
\text { Ansatzstelle } \\
\text { bis zur } \\
\text { Haarpapille }\end{array}$ & & \\
\hline \multicolumn{2}{|l|}{ a } & $42^{\circ} 43^{\prime}$ & $0.531 \mathrm{~mm}$ & $0.413 \mathrm{~mm}$ & $0.118 \mathrm{~mm}$ & $0.360 \mathrm{~mm}$ & $0.280 \mathrm{~mm}$ \\
\hline \multicolumn{2}{|l|}{ b } & $45^{\circ}$ & 0.565 & 0.395 & 0.170 & 0.400 & 0.279 \\
\hline \multicolumn{2}{|l|}{ c } & $45^{\circ}$ & 0.508 & 0.395 & 0.113 & 0.359 & 0.279 \\
\hline \multicolumn{2}{|l|}{$c^{\prime}$} & $43^{\circ} 24^{\prime}$ & 0.407 & - & - & 0.280 & - \\
\hline \multicolumn{2}{|l|}{ d } & $45^{\circ}$ & 0.565 & 0.508 & 0.057 & 0.400 & 0.359 \\
\hline \multicolumn{2}{|l|}{$\mathbf{e}$} & $46^{\circ}$ & 0.556 & 0.389 & 0.167 & 0.400 & 0.280 \\
\hline \multicolumn{2}{|l|}{$\mathbf{e}^{\prime}$} & $49^{\circ} 37^{\prime}$ & 0.523 & 0.365 & 0.158 & 0.398 & 0.278 \\
\hline \multicolumn{2}{|l|}{$\mathbf{f}$} & $47^{\circ} \quad 1^{\prime}$ & 0.601 & 0.492 & 0.109 & 0.440 & 0.360 \\
\hline \multicolumn{2}{|l|}{ g } & $47^{\circ} 37^{\prime}$ & 0.542 & 0.488 & 0.054 & 0.400 & 0.360 \\
\hline \multicolumn{2}{|l|}{$g^{\prime}$} & $51^{\circ} 47^{\prime}$ & 0.458 & - & - & 0.360 & - \\
\hline \multicolumn{2}{|l|}{$\mathrm{g}^{\prime \prime}$} & $63^{\circ} 26^{\prime}$ & 0.447 & - & - & 0.400 & 一 \\
\hline \multicolumn{2}{|l|}{$\mathbf{h}$} & $48^{\circ} \quad 8^{\prime}$ & 0.645 & 0.538 & 0.107 & 0.480 & 0.401 \\
\hline \multicolumn{2}{|l|}{$\mathbf{i}$} & $48^{\circ} 49^{\prime}$ & 0.638 & 0.638 & 0 & 0.480 & 0.480 \\
\hline \multicolumn{2}{|l|}{$\mathbf{j}$} & $49^{\circ} \cdot 11^{\prime}$ & 0.582 & 0.529 & 0.053 & 0.440 & 0.400 \\
\hline \multicolumn{2}{|l|}{$\mathbf{k}$} & $49^{\circ} 56^{\prime}$ & 0.574 & 0.417 & 0.157 & 0.439 & 0.319 \\
\hline \multicolumn{2}{|l|}{1} & $49^{\circ} 56^{\prime}$ & 0.574 & 0.522 & 0.052 & 0.439 & 0.399 \\
\hline \multicolumn{2}{|l|}{$\mathrm{m}$} & $50^{\circ} 11^{\prime}$ & 0.469 & 0.469 & 0 & 0.360 & 0.360 \\
\hline \multicolumn{2}{|l|}{$\mathrm{m}^{\prime}$} & $58^{\circ} 34^{\prime}$ & 0.422 & - & 一 & 0.360 & - \\
\hline \multicolumn{2}{|l|}{$\mathbf{n}$} & $50^{\circ} \quad 42^{\prime}$ & 0.568 & 0.516 & 0.052 & 0.440 & 0.399 \\
\hline \multicolumn{2}{|l|}{0} & $51^{\circ} 38^{\prime}$ & 0.612 & 0.510 & 0.102 & 0.480 & 0.400 \\
\hline \multicolumn{2}{|l|}{$\sigma^{\prime}$} & $53^{\circ} 54^{\prime}$ & 0.594 & 0.495 & 0.099 & 0.480 & 0.400 \\
\hline \multicolumn{2}{|l|}{$\mathbf{p}$} & $51^{\circ} 41^{\prime}$ & 0.510 & 0.459 & 0.051 & 0.400 & 0.360 \\
\hline \multicolumn{2}{|l|}{$p^{\prime}$} & $59^{\circ} \quad 3^{\prime}$ & 0.466 & - & - & 0.400 & - \\
\hline \multicolumn{2}{|l|}{$\mathrm{p}^{\prime \prime}$} & $56^{\circ} 58^{\prime}$ & 0.478 & 一 & - & 0.401 & - \\
\hline$q$ & & $52^{\circ} 19^{\prime}$ & 0.556 & 0.404 & 0.152 & 0.440 & 0.320 \\
\hline $\mathbf{r}$ & & $55^{\circ} 43^{\prime}$ & 0.533 & 0.436 & 0.097 & 0.440 & 0.360 \\
\hline $\mathbf{r}^{\prime}$ & & $49^{\circ} 11^{\prime}$ & $0.58 \dot{2}$ & 一 & - & 0.440 & - \\
\hline Durch- & $\mathbf{H}$ & $48^{\circ} 42^{\prime}$ & 0.563 & & . & 0.422 & 0.355 \\
\hline & $\mathbf{N}$ & $53^{\circ} 59^{\prime}$ & 0.486 & & : & 0.391 & 0.339 \\
\hline
\end{tabular}


Tabelle 1.0. Unterschenkel (Streckseite).

\begin{tabular}{|c|c|c|c|c|c|c|c|}
\hline \multirow{2}{*}{\multicolumn{2}{|c|}{$\underset{\text { nummer }}{\text { Haar- }}$}} & \multirow{2}{*}{$\begin{array}{c}\text { nicht } \\
\text { korrigierter } \\
\text { Haut-Haar- } \\
\text { wurzel- } \\
\text { Winkel (ग) }\end{array}$} & \multirow{2}{*}{$\begin{array}{l}\text { Haar- } \\
\text { wurzel- } \\
\text { länge }\end{array}$} & \multicolumn{2}{|c|}{$\begin{array}{l}\text { Ansatzhöhe des Arrek- } \\
\text { tors an Haarwurzel }\end{array}$} & \multirow[b]{2}{*}{$\mathbf{E P}^{\prime}$} & \multirow[b]{2}{*}{$\mathbf{E M}^{\prime}$} \\
\hline & & & & $\begin{array}{c}\text { Iänge vom } \\
\text { Epithel bis } \\
\text { zur Ansatz } \\
\text { stelle }\end{array}$ & $\begin{array}{c}\text { Länge von } \\
\text { Ansatzstelle } \\
\text { bis zur } \\
\text { Haarpapille }\end{array}$ & & \\
\hline \multicolumn{2}{|l|}{$\mathbf{a}$} & $33^{\circ} 9^{\prime}$ & $0.586 \mathrm{~mm}$ & $0.513 \mathrm{~mm}$ & $0.073 \mathrm{~mm}$ & $0.320 \mathrm{~mm}$ & $0.281 \mathrm{~mm}$ \\
\hline \multicolumn{2}{|l|}{$a^{\prime}$} & $34^{\circ} 2 \alpha^{\prime}$ & 0.496 & - & - & 0.280 & - \\
\hline \multicolumn{2}{|l|}{$a^{\prime \prime}$} & $36^{\circ} 52^{\prime}$ & 0.400 & - & - & 0.240 & - \\
\hline \multicolumn{2}{|l|}{ b } & $36^{\circ} 52^{\prime}$ & 0.600 & 0.467 & 0.133 & .0 .360 & 0.280 \\
\hline \multicolumn{2}{|l|}{$\mathbf{b}^{\prime}$} & $44^{\circ} 13^{\prime}$ & 0.516 & - & - & 0.360 & - \\
\hline \multicolumn{2}{|l|}{ c } & $37^{\circ} \quad 3^{\prime}$ & 0.665 & 0.665 & 0 & 0.401 & 0.401 \\
\hline \multicolumn{2}{|l|}{ d } & $37^{\circ} 27^{\prime}$ & 0.592 & 0.592 & 0 & 0.360 & 0.360 \\
\hline \multicolumn{2}{|l|}{$\mathbf{e}$} & $37^{\circ} 34^{\prime}$ & 0.525 & 0.460 & 0.066 & 0.320 & 0.280 \\
\hline \multicolumn{2}{|l|}{$\mathrm{e}^{\prime}$} & $45^{\circ} 53^{\prime}$ & 0.446 & 0.390 & 0.056 & 0.320 & 0.280 \\
\hline \multicolumn{2}{|l|}{$e^{\prime \prime}$} & $39^{\circ} 37^{\prime}$ & 0.376 & - & - & 0.240 & - \\
\hline \multicolumn{2}{|l|}{ f } & $37^{\circ} 58^{\prime}$ & 0.519 & 0.454 & 0.065 & 0.319 & 0.279 \\
\hline \multicolumn{2}{|l|}{$\mathbf{g}$} & $38^{\circ} \quad 6^{\prime}$ & 0.647 & 0.517 & 0.130 & 0.399 & 0.319 \\
\hline \multicolumn{2}{|l|}{$\mathbf{h}$} & $39^{\circ} 56^{\prime}$ & 0.561 & 0.549 & 0.012 & 0.360 & 0.352 \\
\hline \multicolumn{2}{|l|}{$\mathbf{h}^{\prime}$} & $46^{\circ} 38^{\prime}$ & 0.495 & - & - & 0.360 & - \\
\hline \multicolumn{2}{|l|}{ i } & $41^{\circ} 21^{\prime}$ & 0.667 & 0.485 & 0.182 & 0.441 & 0.320 \\
\hline \multicolumn{2}{|l|}{ j } & $48^{\circ} 22^{\prime}$ & 0.481 & 0.427 & 0.054 & 0.359 & 0.319 \\
\hline \multicolumn{2}{|l|}{$j^{\prime}$} & $45^{\circ}$ & 0.452 & - & - & 0.320 & - \\
\hline \multicolumn{2}{|l|}{$j^{\prime \prime}$} & $49^{\circ} 16^{\prime}$ & 0.475 & - & - & 0.360 & - \\
\hline \multicolumn{2}{|l|}{$\mathbf{k}$} & $48^{\circ} 27^{\prime}$ & 0.588 & 0.481 & 0.107 & 0.440 & 0.360 \\
\hline \multirow{2}{*}{$\begin{array}{l}\text { Durch- } \\
\text { schnitt }\end{array}$} & $\mathbf{H}$ & $39^{\circ} 40^{\prime}$ & 0.585 & & & 0.371 & 0.323 \\
\hline & $\mathrm{N}$ & $42^{\circ} 44^{\prime}$ & 0.457 & & & 0.310 & 0.280 \\
\hline
\end{tabular}


Tabelle 11. Unterschenkel (Beugeseite).

\begin{tabular}{|c|c|c|c|c|c|c|c|}
\hline \multirow{2}{*}{ Huar- } & & \multirow{2}{*}{$\begin{array}{c}\text { nicht } \\
\text { korrigierter } \\
\text { Hant-Haar- } \\
\text { wurzel- } \\
\text { Winkel (l.) }\end{array}$} & \multirow{2}{*}{$\begin{array}{l}\text { Haar- } \\
\text { wurzel- } \\
\text { länge }\end{array}$} & \multicolumn{2}{|c|}{$\begin{array}{l}\text { Ansatzhöhe des Arrek- } \\
\text { tors an Haarwurzel }\end{array}$} & \multirow[b]{2}{*}{$\mathbf{E P} \mathbf{P}^{\prime}$} & \multirow[b]{2}{*}{ EMI' } \\
\hline & & & & $\begin{array}{c}\text { Iänge vom } \\
\text { Epithel bis } \\
\text { zur Ansatz } \\
\text { stelle }\end{array}$ & $\begin{array}{c}\text { Iänge von } \\
\text { Ansatzstelle } \\
\text { bis zur } \\
\text { Haarpapille }\end{array}$ & & \\
\hline \multicolumn{2}{|l|}{ a } & $36^{\circ} 50^{\prime}$ & $0.867 \mathrm{~mm}$ & $0.800 \mathrm{~mm}$ & $0.067 \mathrm{~mm}$ & $0.520 \mathrm{~mm}$ & $0.480 \mathrm{~mm}$ \\
\hline \multicolumn{2}{|l|}{$a^{\prime}$} & $42^{\circ} 43^{\prime}$ & 0.649 & - & - & 0.440 & - \\
\hline \multicolumn{2}{|l|}{ b } & $39^{\circ} \quad 6^{\prime}$ & 0.825 & 0.635 & 0.190 & 0.520 & 0.400 \\
\hline \multicolumn{2}{|l|}{$b^{\prime}$} & $41^{\circ} 59^{\prime}$ & 0.538 & 0.418 & 0.120 & 0.360 & 0.280 \\
\hline \multicolumn{2}{|l|}{$b^{\prime \prime}$} & $42^{\circ} 25^{\prime}$ & - & - & - & - & - \\
\hline \multicolumn{2}{|l|}{ c } & $39^{\circ} 10^{\prime}$ & 0.696 & 0.696 & 0 & 0.440 & 0.440 \\
\hline \multicolumn{2}{|l|}{ d } & $45^{\circ}$ & 0.734 & 0.564 & 0.170 & 0.519 & 0.399 \\
\hline \multicolumn{2}{|l|}{ e } & $45^{\circ}$ & 0.678 & 0.508 & 0.170 & 0.479 & 0.359 \\
\hline \multicolumn{2}{|l|}{$\mathrm{f}$} & $47^{\circ} 17^{\prime}$ & 0.708 . & 0.545 & 0.163 & 0.520 & 0.400 \\
\hline \multicolumn{2}{|l|}{$f^{\prime}$} & $51^{\circ} 30^{\prime}$ & 0.563 & 0.512 & 0.051 & 0.441 & 0.401 \\
\hline \multicolumn{2}{|l|}{ g } & $48^{\circ} 33^{\prime}$ & 0.854 & 0.641 & 0.213 & 0.640 & 0.480 \\
\hline \multicolumn{2}{|l|}{$g^{\prime}$} & $54^{\circ} 28^{\prime}$ & 0.737 & - & - & 0.600 & - \\
\hline \multicolumn{2}{|l|}{$\mathrm{h}$} & $50^{\circ} 14^{\prime}$ & 0.729 & 0,521 & 0.208 & 0.560 & 0.400 \\
\hline \multicolumn{2}{|l|}{$\mathrm{h}^{\prime}$} & $50^{\circ} 11^{\prime}$ & 0.625 & - & - & 0.480 & - \\
\hline \multicolumn{2}{|l|}{$\mathrm{h}^{\prime \prime}$} & $53^{\circ} 7^{\prime}$ & - & - & - & - & - \\
\hline \multicolumn{2}{|l|}{$\mathrm{i}$} & $50^{\circ} 54^{\prime}$ & 0.825 & 0.670 & 0.155 & 0.640 & 0.520 \\
\hline \multicolumn{2}{|l|}{$i^{\prime}$} & $59^{\circ} \quad 3^{\prime}$ & 0.699 & 0.512 & 0.187 & 0.599 & 0.439 \\
\hline \multicolumn{2}{|l|}{$j$} & $51^{\circ} 37^{\prime}$ & 0.664 & 0.460 & 0.204 & 0.520 & 0.360 \\
\hline \multicolumn{2}{|l|}{$j$} & $56^{\circ} 39^{\prime}$ & 0.622 & - & - & 0.520 & - \\
\hline \multicolumn{2}{|l|}{$\mathbf{k}$} & $53^{\circ} 7^{\prime}$ & 0.850 & 0.650 & 0.200 & 0.680 & 0.520 \\
\hline \multicolumn{2}{|l|}{1} & $56^{\circ} 58^{\prime}$ & 0.716 & 0.525 & 0.191 & 0.600 & 0.440 \\
\hline \multirow{2}{*}{$\begin{array}{l}\text { Durch- } \\
\text { schnitt }\end{array}$} & $\mathrm{H}$ & $46^{\circ} 59^{\prime}$ & 0.762 & & & 0.553 & 0.433 \\
\hline & $N$ & $50^{\circ} 14^{\prime}$ & 0.633 & & & 0.491 & 0.373 \\
\hline
\end{tabular}




\section{1) Haut-Haarwurzel-Winkel.}

Die Grösse des Haut-Haarwurzel-Winkels ist beträchtlich verschieden, wie in Tabelle 1-11 gezeigt. Der kleinste Winkel ist am Scheitel (ca. $26^{\circ} 34^{\prime}$ ) und der grösste an der Streckseite des Vorderárms (ca. $\left.60^{\circ} 15^{\prime}\right)$ zu finden.

Der Haut-Haarwurzel-Winkel variiert' auch in einem bestimmten Körperteile, aber die Schwankung ist nicht so auffallend als dieselbe der Winkelgrösse in den ganzen untersuchten Körperteilen. Die grösste Differenz zwischen dem grössten und kleinsten Winkel wird an der Beugeseite des Unterschenkels $\left(20^{\circ} 8^{\prime}\right)$ und die kleinste am Gesäss ( $6^{\circ}$ $50^{\prime}$ ) beobachtet.

Die Mittelwerte dieses Winkels in verschiedenen Körperteilen sind in Tabelle 12 dargestellt.

Tabelle 12. Haut-Haarwurzel-Winkel.

\begin{tabular}{|c|c|c|c|c|}
\hline \multirow[b]{2}{*}{ Körperteile } & \multicolumn{2}{|c|}{ Haupthaar } & \multicolumn{2}{|c|}{ Nebenhaar } \\
\hline & $\begin{array}{l}\text { Zahl der unter- } \\
\text { suchten Haare }\end{array}$ & $\begin{array}{l}\text { Winkel } \\
\left(\mathbf{M}^{\prime} \pm \sigma^{\prime}\right)\end{array}$ & $\begin{array}{l}\text { Zahl der unter- } \\
\text { suchten Haare }\end{array}$ & $\begin{array}{l}\text { Winkel } \\
\left(\mathbf{M}^{\prime} \pm \sigma^{\prime}\right)\end{array}$ \\
\hline $\begin{array}{c}\text { Vorderarm } \\
\text { (Streckseite) }\end{array}$ & 9 & $52^{\circ} 53^{\prime} \pm 3^{\circ} 31.5^{\prime}$ & 4 & $58^{\circ} 51^{\prime} \pm 3^{\circ} 10.8^{\prime}$ \\
\hline $\begin{array}{c}\text { Oberarm } \\
\text { (Streckseite) }\end{array}$ & 15 & $48^{\circ} 58^{\prime} \pm 2^{\circ} 38.3^{\prime}$ & 7 & $56^{\circ} 20^{\prime} \pm 5^{\circ} 24.2^{\prime}$ \\
\hline $\begin{array}{l}\text { Oberschenkel } \\
\text { (Lateralseite) }\end{array}$ & 18 & $48^{\circ} 42^{\prime} \pm 2^{\circ} 50.6^{\prime}$ & 9 & $53^{\circ} 59^{\prime} \pm 5^{\circ} 43.4^{\prime}$ \\
\hline $\begin{array}{l}\text { Unterschenkel } \\
\text { (Beugeseite) }\end{array}$ & 12 & $46^{\circ} 59^{\prime} \pm 5^{\circ} 55.5^{\prime}$ & 9 & $50^{\circ} 14^{\prime} \pm 6^{\circ} 4.6^{\prime}$ \\
\hline $\begin{array}{l}\text { Vorderarm } \\
\text { (Beugeseite) }\end{array}$ & 11 & $40^{\circ} 31^{\prime} \pm 4^{\circ} 3.1^{\prime}$ & 1 & $48^{\circ} 22^{\prime}$ \\
\hline $\begin{array}{l}\text { Unterschenkel } \\
\text { (Streckseite) }\end{array}$ & 11 & $39^{\circ} 40^{\prime} \pm 4^{\circ} 32.9^{\prime}$ & 8 & $42^{\circ} 44^{\prime} \pm 4^{\circ} 52.3^{\prime}$ \\
\hline Rücken & 7 & $38^{\circ} 21^{\prime} \pm 2^{\circ} 34.7^{\prime}$ & 4 & $41^{\circ} 41^{\prime} \pm 7^{\circ} 2.4^{\prime}$ \\
\hline Gesäss & 10 & $38^{\circ} 26^{\prime} \pm 2^{\circ} 0.8^{\prime}$ & 11 & $43^{\circ} 17^{\prime} \pm 4^{\circ} 35.6^{\prime}$ \\
\hline Brust & 8 & $37^{\circ} 51^{\prime} \pm 3^{\circ} 15.6^{\prime}$ & 9 & $43^{\circ} 12^{\prime} \pm 3^{\circ} 16.1^{\prime}$ \\
\hline Stirn & 10 & $32^{\circ} 46^{\prime} \pm 2^{\circ} 19.3^{\prime}$ & 1 & $44^{\circ} 17^{\prime}$ \\
\hline Scheitel & 12 & $31^{\circ} 32^{\prime} \pm 3^{\circ} 28.1^{\prime}$ & 1 & $44^{\circ} 7^{\prime}$ \\
\hline
\end{tabular}

$M^{\prime}$ und $\sigma^{\prime}$ sind aus dem nicht korrigierten Winkel (2.) ausgerechnet.

Aus dieser Tabelle ersieht man, dass der Mittelwert des Haut-Haarwurzel-Winkels auch nach den Körperteilen erheblich schwankt, so ist er unter den 11 Körperteilen am grössten (durchschnittlich $52^{\circ} 53^{\prime}\left(\sigma^{\prime}=3^{\circ}\right.$ $\left.31.5^{\prime}\right)$ ) an der Streckseite des Vorderarms, dann folgen die Streckseite des Oberarms (durchschnittlich $48^{\circ} 58^{\prime}\left(\sigma^{\prime}=2^{\circ} 38.3^{\prime}\right)$ ), die Lateralseite des 
Oberschenkels (durchschnittlich $48^{\circ} 42^{\prime} \quad\left(\sigma^{\prime}=2^{\circ} 50.6^{\prime}\right)$ ) u.s.w. und am kleinsten ist er am Scheitel (durchschnittlich $31^{\circ} 32^{\prime}\left(\sigma^{\prime}=3^{\circ} 28.1^{\prime}\right)$ ).

Noch erkennt man aus Tabelle 12, dass in allgemeinen am Kopf die Neigung der Haarwurzel am grössten d.h. der Haut-HaarwurzelWinkel hier am kleinsten ist, damn folgt der Rumpf und an den Extremitäten ist die Neigung der Haarwurzel am kleinsten d.h. der Haut-Haarwurzel-Winkel am grössten.

Vergleicht man feruer den betreffenden Winkel der Haupthaare und den der Nebenhaare miteinander, so kann man finden, dass in allen untersuchten Körperteilen der Winkel der Nebenhaare fast immer grösser als der von Haupthaaren ist.

2) Haarwurzellänge.

Aus Tabelle 1-11 kann man ersehen, dass arn Scheitel und an der Stirn zwei Arten von Haarwurzeln in Bezug auf ihre Länge vorhanden sind, eine lange und eine kurze ; die letztere kommt seltener als die erstere vor. An jedem anderen Körperteile ist auch die Wurzellänge verschieden gross, aber der Unterschied ist nicht so deutlich wie zwischen den zwei obigen Arten am Kopf.

Wenn ich die durchschnittliche Haarwurzellänge an allen untersuchten Körperteilen tabellarisch zusammenstelle, so ergibt sich :

Tabelle 13. Haarwurzellänge.

\begin{tabular}{|c|c|c|c|c|}
\hline \multirow[b]{2}{*}{ Körperteile } & \multicolumn{2}{|c|}{ Haupthaar } & \multicolumn{2}{|c|}{ Nebenhaar } \\
\hline & $\begin{array}{l}\text { Zahl der unter- } \\
\text { suchten Haare }\end{array}$ & $\begin{array}{c}\text { Durchschnitts- } \\
\text { länge } \\
(\mathbf{M} \pm \sigma)\end{array}$ & $\begin{array}{l}\text { Zahl der unter- } \\
\text { suchten Haare }\end{array}$ & $\begin{array}{c}\text { Durchschnitts- } \\
\text { länge } \\
(\mathrm{M} \pm \sigma)\end{array}$ \\
\hline Scheitel & $\begin{array}{l}\mathbf{9} \\
\mathbf{3}\end{array}$ & $\begin{array}{l}2.166 \pm 0.0949 \mathrm{~mm} \\
0.751 \pm 0.0852\end{array}$ & 1 & $0.804 \mathrm{~mm}$ \\
\hline Stirn & $\begin{array}{r}6 \\
\quad 4 \\
\end{array}$ & $\begin{array}{l}1.004 \pm 0.1810 \\
0.681 \pm 0.1130\end{array}$ & 1 & 0.559 \\
\hline Gesäss & 10 & $0.781 \pm 0-0655$ & 11 & $0.684 \pm 0.0588$ \\
\hline $\begin{array}{c}\text { Unterschenkel } \\
\text { (Beugeseite) }\end{array}$ & 12 & $0.762 \pm 0.0726$ & 7 & $0.633 \pm 0.0651$ \\
\hline $\begin{array}{c}\text { Vorderarm } \\
\text { (Streckseite) }\end{array}$ & 9 & $0.689 \pm 0.1031$ & 4 & $0.597 \pm 0.0255$ \\
\hline Brust & 8 & $0.673 \pm 0.0916$ & 9 & $0.572 \pm 0.1091$ \\
\hline Rücken & 7 & $0.631 \pm 0.0736$ & 4 & $0.538 \pm 0.0520$ \\
\hline $\begin{array}{c}\text { Vorderarm } \\
\text { (Beugeseite) }\end{array}$ & 11 & $0.596 \pm 0.0767$ & 1 & 0.481 \\
\hline $\begin{array}{l}\text { Unterschenkel } \\
\text { (Streckseite) }\end{array}$ & 11 & $0.585 \pm 0,0578$ & 8 & $0.457 \pm 0.0459$ \\
\hline $\begin{array}{l}\text { OberschenkeI } \\
\text { (I,ateralseite) }\end{array}$ & 18 & $0.563 \pm 0.0435$ & 9 & $0.486 \pm 0.0627$ \\
\hline $\begin{array}{c}\text { Oberarm } \\
\text { (Streckseite) }\end{array}$ & 15 & $0.548 \pm 0.0527$ & 7 & $0.516 \pm 0.0437$ \\
\hline
\end{tabular}


In den 11. untersuchten Körperteilen ist also die Haarwurzellänge des Haupthaares im allgemeinen am Scheitel am längsten (durchschnittlich $2.166 \mathrm{~mm}(\sigma=0.0949 \mathrm{~mm})$ ), dann folgen die Stirn (durchschnittlich 1.004 $\mathrm{mm}(\sigma=0.1810 \mathrm{~mm})$ ), das Gesäss (durchschnittlich $0.781 \mathrm{~mm}(\sigma=0.0655$ $\mathrm{mm})$ ), die Beugeseite des Unterschenkels (durchschnittlich $0.762 \mathrm{~mm}$ $(\sigma=0.0726 \mathrm{~mm}))$ u. s. w., und ist an der Streckseite des Oberarms (durchschnittlich $0.548 \mathrm{~mm}(\sigma=0.0527 \mathrm{~mm}))$ am kürzesten.

Wie schon angegeben, findet sich am Kopf (Scheitel und Stirn) eine geringe Zahl von Haaren mit kurzer Wurzellänge, die mit der Haarwurzel an anderen Körperteilen (Rumpf und Extremitäten) verglichen keinen merklichen Längenunterschied zeigen; dennoch kann man wohl sagen, dass im allgemeinen am Kopf die Haarwurzel beträchtlich länger ist als bei anderen Körperteilen, wo die Wurzellänge überall fast gleich gross ist.

Über die Haarwurzellänge des Erwachsenen haben bis jetzt einige Autoren Mitteilungen gebracht. Nach Rauber (10) beträgt die Haarbalglänge des längeren Haares $2.7 \mathrm{~mm}$ bis $3.8 \mathrm{~mm}$, nach A u burtin (4) 2.68 $\mathrm{mm}-3.3 \mathrm{~mm}$, nach Kanaizuka (6) am Scheitel $2.55 \mathrm{~mm}-3.89 \mathrm{~mm}$, an der Stirn $3.57 \mathrm{~mm}-4.35 \mathrm{~mm}$. Die Haarwurzellänge ist am Scheitel des Erwachsenen nach Kanaizuka durchschnittlich ca, $3.252 \mathrm{~mm}$ und nach mir beim Neugeborenen $2.166 \mathrm{~mm}$. So erkennt man, dass die Haarwurzellänge am Scheitel beim Neugeborenen durchschnittlich ca. 1 mm kürzer als beim Erwachsenen ist.

3) Ansatzhöhe des Muskels.

Zur Bestimmung der Muskelansatzhöhe am Hauptbaar babe ich die ganze Haarwurzellänge in 6 gleichgrosse Abschnitte geteilt und diese Klassen von der Hautoberfläche an als a, b, c, d, e, f und die Klassengrenzen als $1,2,3,4,5,6,7$ bezeichnet.

Dann habe ich die Mittelwerte der Muskelansatzhöhe in allen untersuchten Körperteilen nach der Methode von Adachi ausgerechnet und in folgender Tabelle dargelegt.

Aus dieser Tabelle kann man seben, dass der Arrektor niemals am Abschnitt a oder b, nämlich am oberen Drittel der Haarwurzel sich ansetzt.

Ferner setzt sich der Arrektor im Scheitel am häufigsten am Abschnitt c an, also am oberen Teil des mittleren Drittels. Daher behaupte ich mit Sicherheit, dass die Ansatzstelle des Arrektors am Scheitel meistens viel höher als in der mittleren Höhe der Haarwurzel liegt, wie von Häggqvist (5) und Kanaizuka (6) schon geäussert wurde, während er nach Unna (12) ain mittleren Abschnitt der Haarwurzel und nach Bonnet (1) an ihrem unteren Drittel inseriert. Aber dadurch, dass an 


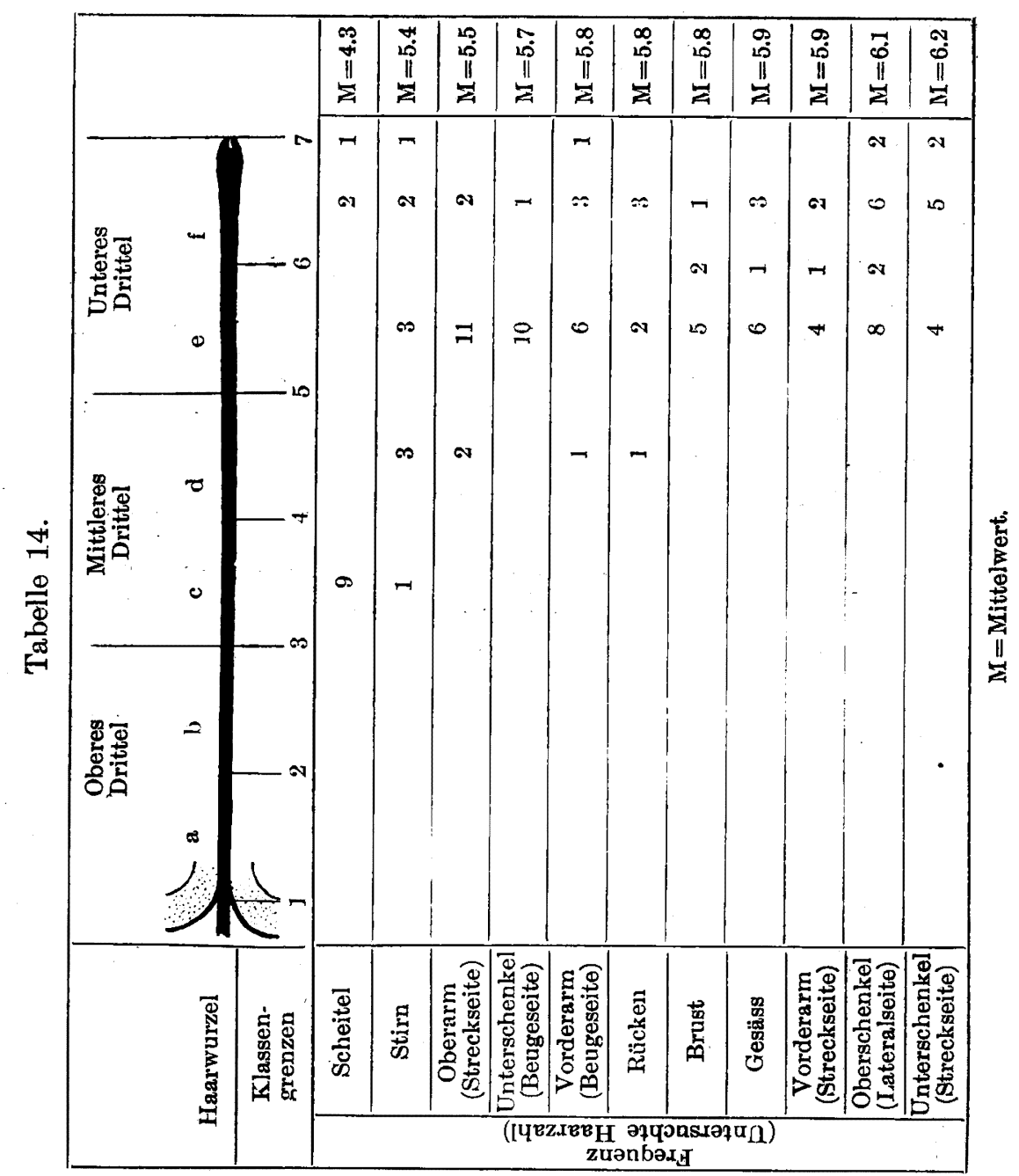

den 3 Fällen unter 12 untersuchten Haarwurzeln des Scheitels der Muskel sehr niedrig sich ansetzt, scheint die durchschnittliche Ansatzhöhe (Mittelwert 4.3) am mittleren Abschnitt zu liegen, wie von Unna beschrieben wurde.

An der Stiru setzt der Arrektor sich gewöhnlich am Abschnitt d oder e, also meistens in der Höhe des unteren Drittels der Haarwurzel an, aber die durchschnittliche Ansatzhōhe (Mittelwert 5.4) liegt etwas unterhalb des unteren Drittels.

An anderen Körperteilen inseriert der Arrektor im allgemeinen am 
Abschnitt e oder f, also liegt die Ansatzhöhe am unteren Drittel, wie es Bonnet bereits beschrieben hatte, und die durchschnittliche Ansatzhöhe steht auch ungefähr in der Mitte des unteren Drittels, aber nur an der Streckseite des Oberarms etwas höher.

In Tabelle 1-11 ist die Muskelansatzhöhe am Haarbalg und die vertikale Tiefe vom Epithel bis zur Ansatzstelle sowie bis zur Haarpapille jedes Haares angegeben.

Weiter über die Vergleichung der Muskelansatzstelle am Haarbalg der Haupt- und Nebenhaaren beschreibt Kanaizuka neuerdings in seiner Arbeit: „Nach Rabl (11) setzt der Arrektor immer mit seinem tiefsten Zipfel am Mittelhaar und den geteilten Zipfeln an den Nebenhaaren an, so liegt seine Ansatzstelle an den Nebenhaaren immer viel oberflächlicher als die des Mittelhaares. Aber mein Befund am Wachsplattenmodelle deckt sich nicht immer mit demselben von Rabl. Ich konnte einen Arrektor sehen, der mit seinem tiefsten Zipfel am Nebenhaar ansetzt, so dass hier die Sache ganz umgekehrt sich verhält zur eben angegebenen allgemeinen Regel." Ich habe nach der mikrogeometrischen Messung an den Haupt- und Nebenhaaren dasselbe Verhältnis festgestellt, wie Kanaizuka, dass die Muskelansatzstelle der Nebenhaare nicht immer oberflächlicher als die der Haupthaare steht.

So wie in obigen Tabellen 1-11 gezeigt, unter den 12 Haargruppen konnte ich 3 Haargruppen vorfinden, an welchen der Arrektor sich mit seinem tiefsten Zipfel am Haupthaar und mit dem anderen oberflächlicheren Zipfel am Nebenhaar ansetzt (e Gruppe der Lateralseite des Oberschenkels, i und b Gruppe der Beugeseite des Unterschenkels) und in 4 Haargruppen das Verhältnis umgekehrt ist; so setzt sich der Arrektor mit seixem tiefsten Zipfel am Nebenhaar, mit seinem oberflächlicheren Zipfel am Haupthaar an (g Gruppe des Scheitels, a Gruppe der Brust, d Gruppe des Gesässes, f Gruppe der Beugeseite des Unterschenkels). An anderen 5 Haargruppen (i Gruppe des Gesässes, k Gruppe der Streckseite des Oberarms, f Gruppe der Beugeseite des Vorderarms, o Gruppe der Lateralseite des Oberschenkels, e Gruppe der Streckseite des Unterschenkels) fand ich die Muskelansatzstelle am Haarbalg des Haupthaare und Nebenhaare gleich hoch stehend.

\section{Resultate.}

Die Hauptresultate der vorliegenden Studie über den Haut-Haarwurzel-Winkel, die Haarwurzellänge und Muskelansatzhöhe beim japanischen Neugeborenen lassen sich folgendermassen zusammenfassen. 
1) Der grösste Haut-Haarwurzel-Winkel des Haupthaares beträgt an der Streckseite des Vorderarms ca. $60^{\circ} 15^{\prime}$ und der kleinste am Scheitel ca. $26^{\circ} 34^{\prime}$.

2) Die Schwankung der Grösse des Haut-Haarwurzel-Winkels des Haupthaares in jedem bestimmten Körperteile ist viel kleiner als dieselbe der Winkelgrösse in den ganzen untersuchten Körperteilen.

3) Die Grösse des Haut-Haarwurzel-Winkels variiert nach den Körperteilen in hohem Masse, wie Tabelle 12 zeigt. In den 11 untersuchten Körperteilen ist der Winkel an der Streckseite des Vorderarms am grössten $\left(M^{\prime}=52^{\circ} 53^{\prime}\left(\sigma^{\prime}=3^{\circ} 31.5^{\prime}\right)\right)$ und am Scheitel am kleinsten $\left(M^{\prime}=31^{\circ} 32^{\prime}\right.$ $\left.\left(\sigma^{\prime}=3^{\circ} 28.1^{\prime}\right)\right)$.

4) Der Haut-Haarwurzel-Winkel ist in der Regel in den Extremitäten am grössten, dann folgen der Rumpf und er ist im Kopf am kleinsten.

5) Der Haut-Haarwurzel-Wiukel des Haupthaares ist immer kleiner als der des dazu gehörigen Nebenhaares.

6) Es gibt zwei Arten von Haarwurzeln am Kopf, die lange und die kurze Wurzel ; aber in anderen untersuchten Körperteilen ist die Haarwurzel in jedem Körperteile fast gleich lang.

7) Die Haarwurzellänge des Haupthaares an der Stirn (durchschnittlich $1.004 \mathrm{~mm}$ ) ist ca. halb so lang wie die am Scheitel (durchsschnittlich $2.166 \mathrm{~mm}$ ) und in anderen Körperteilen-Rumpf und Extremitäten (durchschnittlich ca. $0.648 \mathrm{~mm}$ )-ist sie überall ungefähr gleich laug, so beträgt sie ca. ein Drittel von derselben des Scheitels (das Verhältnis ist wie $3: 1.5: 1$ ).

8) Die Proportion der Wurzellänge des Haupthaares zwischen dem Neugeborenen und Erwachsenen ist am Scheitel ungefähr $2: 3$.

9) Die Muskelansatzstelle am Haarbalg ist nach den Körperteilen verschieden hoch ; so liegt beim Neugeborenen die Ansatzstelle des Arrektors am Haupthaar im allgemeinen am Scheitel im oberen Teil des mittleren Drittels, an der Stirn in der Näbe des unteren Drittels und an anderen Körperteilen stets in der Nähe der mittleren Höhe des unteren Drittels des Haarbalges.

10) Die Muskelansatzstelle am Nebenhaar ist nicht immer oberflächlicher als am Haupthaar, sondern zuweilen steht die Ansatzstelle der beiderlei Haare in ganz gleicher Höhe oder vielmehr die des Haupthaares viel oberflächlicher als die des Nebenhaares.

Zum Schluss spreche ich Herrn Professor K. Okajima für seine stete liebenswürdige Unterstützung bei dieser Arbeit meinen herzlichsten Dank aus. 


\section{Literatur.}

1) Bonnet, R., Haut und Anhang. Vergleichende Histologie der Haussäugetiere. Herausgegeben von W. Ellenberger. 1887. (Zit. in Möllen dorff's Handbuch der mikroskopischen Anatomie des Menschen. Haut und Sinnesorgane. Berlin 1927).

2) Basler, A., Methoden zur Bestimmung der Wurzelfestigkeit und des Neigungswinkels menschlicher Haare. Abderhalden's Handb. der biolog. Arbeitsmeth. Abt. V, Teil 2, Heft 12. 1929.

3) Frédéric, J., Untersuchungen über die Rassenunterschiede der menschlichen Kopfhaare. Zeitschr. f. Morph. u. Anthr. Band 9. 1906.

4) Fritsch, G. und Auburtin, G., Das Vorkommen von Kolbenhaaren und die Veränderungen derselben beim Haarwiederersatz. Arch. f. mikr. Anat. Bd. 47.

5) Häggqvist, G., Studien über die Temperatursinne der Haut des Menschen. 1915. Kungl. svenska vetenskapsakad. handlingar. Bd. 53, No. 2. 1915.

6) Kanaizuka, Z., Beiträge zur Morphologie des Musculus arrector pili. Folia Anat. Japon. Bd. IV. 1926.

7) Martin, R., Lehrbuch der Anthropologie. Bd. 1. s. $496 . \quad$ Jena 1928.

8) Okajima, K. und Koibuchi, S., Ửber die Haur-Arrektor-Winkel beim japanischen Neugeborenen. Folia Anat. Japon. Bd. X. S. 531-532. 1932.

9) Okajima, K., Mikrogeometrische Messung. Abderhalden's Handb. der biolog. Arbeitsmeth. Abt. V, Teil 2 (2)- 1. 1932.

10) Rauber, A. und Kopsch, Er., Iehrbuch der Anatomie des Menschen. XIII. Aufl. 1930.

11) Rabl, H., Histologie der normalen Haut des Menschen. Mraćek's Handbuch der Hautkrankheiten. 1902.

12) Unna, P., Entwicklungsgeschichte und Anatomie der Haut. Zi e ms en's Handbuch der speziellen Pathologie und Therapie. Bd. 14. 1883. (Zit. bei Möllend or ff). 